\title{
TOMOGRAFIA COMPUTADORIZADA EM PACIENTES PORTADORES DE NEUROCISTICERCOSE COM HIPERTENSÃO INTRACRANIANA POR HIDROCEFALIA OBSTRUTIVA: COMPARAÇÃO COM VENTRICULOGRAFIA COM DIMER-X
}

\author{
BENEDICTO OSCAR COLLI \\ NELSON MARTELLI \\ JOAO ALBERTO ASSIRATI JR. \\ HELIO RUBENS MACHADO \\ ANGELA BELLUCI
}

O acometimento cerebral pela cisticercose apresenta grande importância em nosso meio devido à morbidade e mortalidade elevadas e à sua alta incidência. Entre as diferentes manifestações clínicas da neurocisticercose, destaca-se a hipertensão intracraniana, pela sua gravidade e potencialidade cirúrgica 1,5,13,17,21,24,27,37,38. No Hospital das Clínicas da Faculdade de Medicina de Ribeirão Preto a neurocisticercose foi observada em 2,7\% dos pacientes atendidos por apresentarem quadros neurológicos e a hipertensão intracraniana (HIC), pura ou combinada com outra sintomatologia, foi observada em $35,8 \%{ }^{38}$. Em muitos casos, as reações no líquido cefalorraqueano (LCR) de pacientes com hidrocefalia obstrutiva por neurocisticercose não permitem seu diagnóstico ${ }^{13,17}$, assim como os exames radiológicos simples, a angiografia cerebral e a ventriculografia $2,3,4,5,6,7,10,12,13,15,16,20,21,22,23,24,28,30,32,33,35,37,38,39$. Com o advento da tomografia computadorizada (TC) têm sido descritas alterações típicas da neurocisticercoses, abrindo-se nova perspectiva para sua confirmação $7,8,11,15,18,19,20,22,23,29,31,34,36,39$.

No presente trabalho temos por objetivo relatar as alterações observadas nas TCs de pacientes com hidrocefalia obstrutiva por neurocisticercose, compará-las com os achados ventriculográficos e tentar estabelecer alguns critérios para a indicação do tratamento cirúrgico desses casos.

\section{MATERIAL E METADOS}

Foram revistos os prontuários de 16 pacientes (10 femininos e 6 masculinos), com neurocisticercose que evoluiram com hipertensão intracraniana por hidrocefalia obstrutiva e que foram submetidos a exploração radiológica através de radiografias simples do crânio e TC no periodo de 1979 a 1982, no Hospital das Clínicas da Faculdade de

Trabalho realizado no Departamento de Cirurgia, Ortopedia e Traumatologia (Disciplina de Neurocirurgia) da Faculdade de Medicina de Ribeirão Preto da Universidade de São Paulo, apresentado no XX Congresso Latínoamericano de Neurocirurgia (Săo Paulo, 1983). 
Medicina de Ribeirão Preto; 12 destes pacientes foram também submetidos a ventriculografia com Dimer-X. O diagnóstico de neurocisticercose fol efetuado em 10 pacientes através da positividade da reação de fixação de complemento no LCR; em um, pela observação cirúrgica; em 5, mediante as reações celulares do LCR e dos aspectos ventriculográficos e tomográficos sugestivos da doença.

A técnica de execuçáo das ventriculografias foi referida em trabalho anterior 14. As TCs foram realizadas empregando-se uma unidade Delta-Scan 2010 (Ohio Nuclear Incorporation), com matriz 256x256. Os cortes foram efetuados paralelos à linha órbito-meatal, com intervalo de $10 \mathrm{~mm}$, antes e após a injeção de contraste endovenoso. Em dois casos foram efetuadas TC após a realização da ventriculografia, com a finalidade de se aproveitar o contraste (Dimer-X) no sistema ventricular para a visualização de possíveis massas no seu interior. Na análise dos exames radiológicos foi considerada a época da sua realização em relação às intervençóes cirúrgicas para abordagem direta de cisticercos, e só foram considerados aqueles realizados previamente a elas. Para a comparação entre os resultados ventriculográficos e tomográficos foram utilizados os exames realizados na mesma época.

\section{RESULTADOS}

Na tabela 1 são apresentados os resumos das manifestaçóes clínicas dos pacientes e as principais alterações observadas nos exames neurorradiológicos (RX simples do crânio, ventriculografia e TC).

As radiografias simples do crânio foram normais em 8 casos. Em 7 destes foram observadas alterações atribufdas a hipertensão intracraniana (erosóes selares, impressões digiformes acentuadas e/ou diástase de suturas); em apenas um caso foram observadas pequenas calcificações patológicas associadas a sinais de hipertensão intracraniana.

A TC evidenciou perênquima cerebral normal em 9 casos; as alterações observadas nos 7 restantes foram divididas em 2 grupos: 1 - áreas hipodensas arredondadas, varíáveis em número e tamanho (5 a $30 \mathrm{~mm}$ ), de aspectos geralmente cístico, que podem apresentar ou não um halo captante ao seu redor, observadas em 5 casos (Fig. 1); 2 - pequenas calcificações de formas variadas, únicas ou difusas, no parênquima encefálico, sem localização preferencial, observadas em 6 casos (Fig. 2). Os dois tipos de alterações foram verificados em pacientes com sintomatologia clínica com duraçáo de 3 semanas a 5 anos ou mais. Em dois casos foram observadas áreas hipodensas e calcificaçóes no mesmo exame. A TC mostrou hidrocefalia em todos pacientes que foram submetidos ao exame antes de sofrerem derivação do trânsito do LCR (8 casos), exceto um com obstruçáo, provavelmente, do IV ventrículo por massa cística com halo captante nos bordos (Fig. 3). Entre os casos em que a TC fol realizada após a derivação do trânsito do LCR, o sistema ventricular apresentava-se normal em três e dilatado eem 5 (exames realizados por suspeita de mal funcionamento valvular).

As ventriculografias efetuadas foram analisadas em trabalho anterior $14 \mathrm{e}$, fundamentalmente, possibilitaram a localizaçáo do ponto de obstrução. as características 
da obstrução, a presença de massas ocupando espaço no sistema ventricular e irregularidades no contorno das paredes ventriculares (reaçóes inflamatórias). Em um dos casos em que a TC foi realizada após a ventriculografia com Dimer-X, foi evidenciada massa cística no interior do IV ventrículo (demonstrada também pela ventriculografia), com contraste ao seu redor, embora não tenham sido demonstradas falhas de enchimento irregulares nos ventrículos laterais e III ventrículo, verificadas na ventriculografia.

\section{IABEI A}

\begin{tabular}{|c|c|c|c|c|}
\hline mamianio & MAX rFSTASUES CIINICAS & XE rernin & VINTRICLISOCRAFTA & IMPRCRAFTA COPAUTARRKIIAPA \\
\hline $\begin{array}{c}\text { M.A. } \\
40 \text { anos M } \\
26030678\end{array}$ & $\begin{array}{l}\text { 29/09/80 - Crises convulsivas - con } \\
\text { fussao mental his } 9 \text { meses. } \\
02 / 09 / 80 \text { - DVP }\end{array}$ & $\begin{array}{l}18 / 04 / 80- \\
\text { norral. }\end{array}$ & $\begin{array}{l}\text { 12/11/80 - VVLL dilacados. IIIQ a IVP } \\
\text { ventriculos ráo contrastaram. }\end{array}$ & 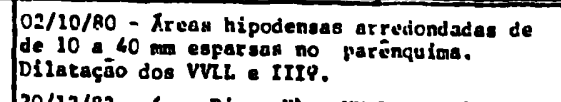 \\
\hline $\begin{array}{c}\text { I.A.S } \\
23 \text { anos } \\
26 \\
080278\end{array}$ & 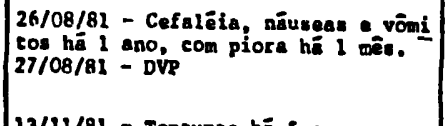 & $\begin{array}{l}\text { 25/10/81 - } \\
\text { aormal. }\end{array}$ & 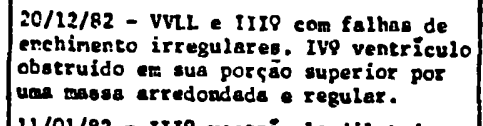 & 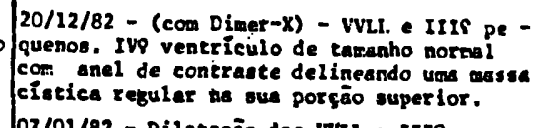 \\
\hline $\begin{array}{l}\text { C.C.S. } \\
45 \text { anos in } \\
26084848\end{array}$ & 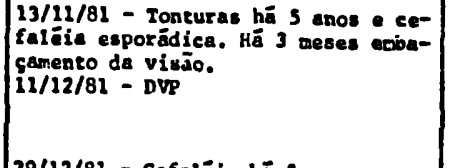 & $\mid \begin{array}{l}25 / 10 / 82- \\
\text { sinain de } \\
\text { HIC }\end{array}$ & $\begin{array}{l}11 / 01 / 82 \text { - III ventriculo dilatado } \\
\text { com falhas de enchimento comunicantes } \\
\text { eatre si e movis (cisticerco racemoso } \\
\text { oot). IVo ventriculo cow falha de en - } \\
\text { ehimento que vai desde o aqueduto ate- } \\
\text { os zecessos laterais. }\end{array}$ & |07/01/82 - Dilatação dos VVLl e IIIP. \\
\hline $\begin{array}{r}\text { J.W.L.G } \\
11 \text { anos } M \\
26087156\end{array}$ & $\begin{array}{l}29 / 12 / 81 \text { - Cefaléia hä } 2 \text { seasanas. } \\
29 / 12 / 81 \text { - DVP } \\
12 / 01 / 82 \text { - CFP - obstrução inflasa- } \\
\text { tốria do forame de Magendie. }\end{array}$ & $\begin{array}{l}29 / 12 / 81- \\
\text { sinais de } \\
\text { HIC }\end{array}$ & $\begin{array}{l}\text { 04/01/82 - VVLL IIIP dilatados. IV } \\
\text { ventr leulo obstruido em sua porgäo su- } \\
\text { perior, irregular, com enchimento par- } \\
\text { cial do recesso lateral esquerdo. }\end{array}$ & $\begin{array}{l}\text { 01/01/82 - Area hipodense de } 1,5 \mathrm{~cm} \text { na tis- } \\
\text { urra inter-hemisferica. } \\
\text { vinl e IIIP notmsis - IV nöo visualizade. }\end{array}$ \\
\hline $\begin{array}{c}13 \text { C.E.S.S } \\
\text { RG } 010752 \\
0.032\end{array}$ & 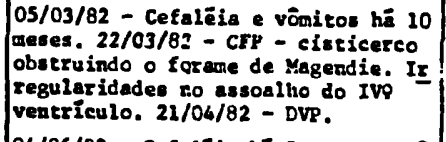 & $\mid \begin{array}{l}08 / 03 / 82- \\
\text { norms! }\end{array}$ & $\begin{array}{l}\text { 08/03/82 - VVLL e IIIR dilatados. IWP } \\
\text { ventriculo obstruido so nivel dos re- } \\
\text { cesaos laterais, coe pequeap contorno } \\
\text { em tega invertida. }\end{array}$ & $\begin{array}{l}\text { 09/03/82 - vVLL diletados , trlo näo visua- } \\
\text { lizado a IVo norwal. }\end{array}$ \\
\hline $\begin{array}{c}\text { K.G.R. } \\
28 \text { anos } E \\
26096351\end{array}$ & $\begin{array}{l}\text { 04/06/82 - Cefaläia hã } 2 \text { meses — vô } \\
\text { niros hê } 3 \text { anos. } \\
11 / 06 / 82 \text { - CFP - cistiterco livre } \\
\text { no IVo ventrículo. }\end{array}$ & $\mid \begin{array}{l}\text { 08/06/82 - } \\
\text { areis de } \\
\text { HIC }\end{array}$ & 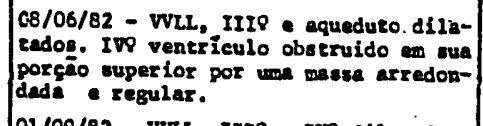 & 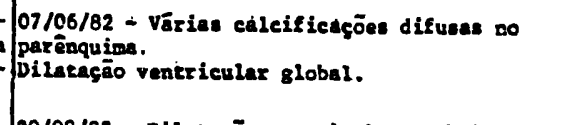 \\
\hline $\begin{array}{l}60 \text { anos } E \\
86020336\end{array}$ & 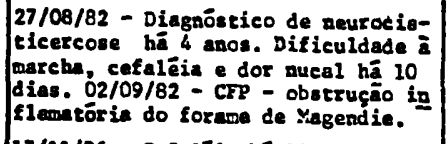 & $\begin{array}{l}30 / 08 / 82- \\
\text { sinais de } \\
\text { uIC }\end{array}$ & 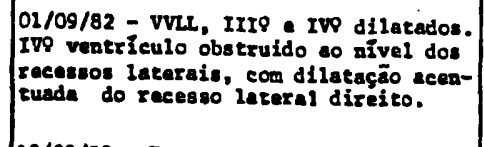 & 30/08/82 - Dilataģ̄o ventericular global. \\
\hline $\begin{array}{l}28 \text {.R. } \\
28 \text { anos } \\
029226\end{array}$ & $\begin{array}{l}\text { 17/11/76 - Cefaläis hä } 30 \text { diss. } \\
22 / 11 / 76 \text { - DVP }\end{array}$ & $\begin{array}{l}19 / 03 / 80- \\
0020101\end{array}$ & $\begin{array}{l}\text { 18/03/80 - Falhas de enchimento irregu } \\
\text { lares no viD. IVo ventrieulo obstrui= } \\
\text { do en gua porçâo ouparior, afilado } \\
\text { terminando em fuado cago. }\end{array}$ & $\left\{\begin{array}{l}23 / 04 / 82=\text { vrl e } 1110 \text { dilatados. IV9 ven- } \\
\text { triculo wä visualizado. }\end{array}\right.$ \\
\hline $\begin{array}{c}24.1 .8 . \\
26036293 \\
2603\end{array}$ & 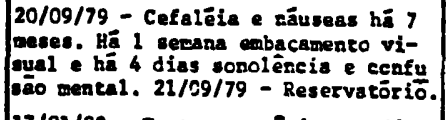 & $\mid \begin{array}{l}21 / 09 / 79- \\
\text { sinsia de } \\
\text { HIC e cal- } \\
\text { cificaçōes. }\end{array}$ & $\begin{array}{l}\text { 29/08/79 - VVLI e IIro dilatados. Oba } \\
\text { truçẫo parcial da porça superior do } \\
\text { queduto por ura nassa arredondada za } \\
\text { gular. }\end{array}$ & $\begin{array}{l}18 / 01 / 80 \text { - Calcificaşöes no parênquima. } \\
\text { Ventrieulos normais. }\end{array}$ \\
\hline $\begin{array}{l}\text { G.T.I. } \\
21 \text { anos } \\
26045523\end{array}$ & 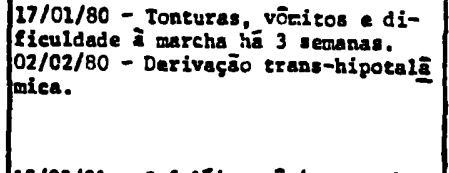 & $\begin{array}{l}24 / 10 / 80- \\
\text { sinais de } \\
\text { HIC }\end{array}$ & 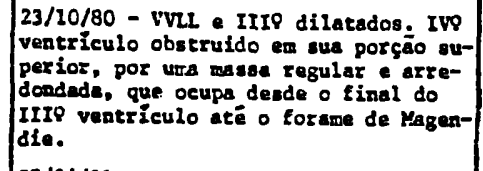 & $\begin{array}{l}18 / 01 / 80 \text { - Calcificaçōes e äreas hipodensas } \\
\text { no parênquima, com retengăo de contraste ca } \\
\text { periferia. } \\
\text { Dilataçäo vearricular globol. }\end{array}$ \\
\hline $\begin{array}{l}28.8 . \\
26069192 \\
062\end{array}$ & 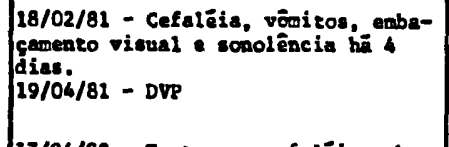 & $\begin{array}{l}11 / 09 / 81- \\
\text { normal }\end{array}$ & 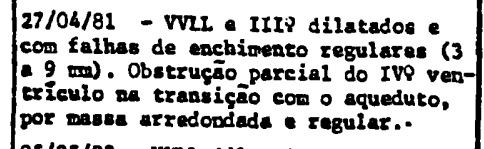 & $11 / 09 / 81-$ noxmal. \\
\hline $\begin{array}{l}23.2 .5 . \\
R G 093592 \\
0.03\end{array}$ & 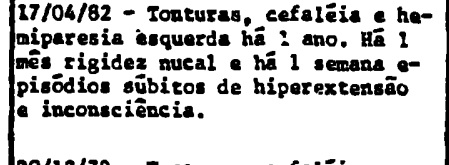 & $\begin{array}{l}22 / 04 / 82- \\
\text { sinais de } \\
\text { HIC }\end{array}$ & 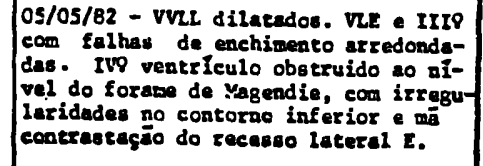 & $\mid$ \\
\hline 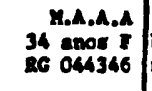 & $\begin{array}{l}\text { 20/12/79 - Touturas, cefalēie e equ- } \\
\text { baçamento visusl hä } 1 \text { ano, con pio- } \\
\text { re hä } 1 \text { wês. 20/02/80 - DVP }\end{array}$ & $\begin{array}{l}21 / 12 / 79- \\
\text { oinais de } \\
\text { HIC }\end{array}$ & & $\begin{array}{l}\text { 27/01/80 - Calcificaçōes no parēnquima. } \\
\text { Dilatagão dos veatriculos laterals — IIro }\end{array}$ \\
\hline $\begin{array}{l}32.8 . \\
32090684 \\
260968\end{array}$ & 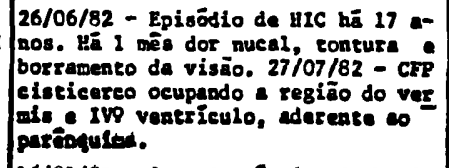 & $\mid$ & & 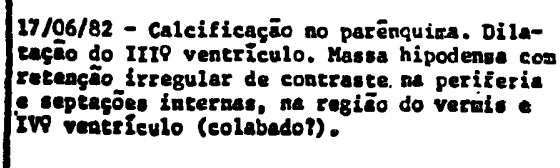 \\
\hline 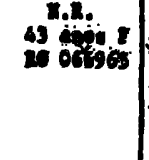 & 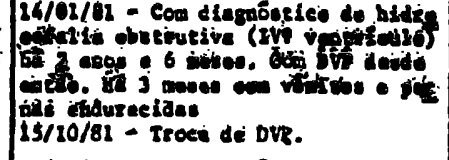 & $\mid \begin{array}{l}25 / 01 / 42 \\
\text { eomit }\end{array}$ & & 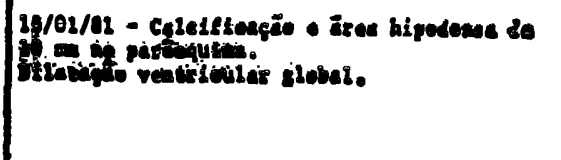 \\
\hline 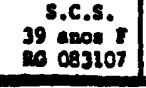 & 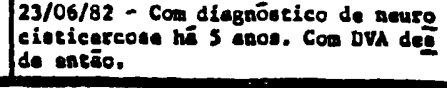 & $\mid \begin{array}{l}29 / 06 / 82- \\
\text { aiseis de } \\
\text { ure }\end{array}$ & & 25/06/02 - Diletegẽo dos Whe - IItP. \\
\hline
\end{tabular}

Tabela 1 - Resumo das observasóes clinicas e resultados dos exames neuroradiologicos. 


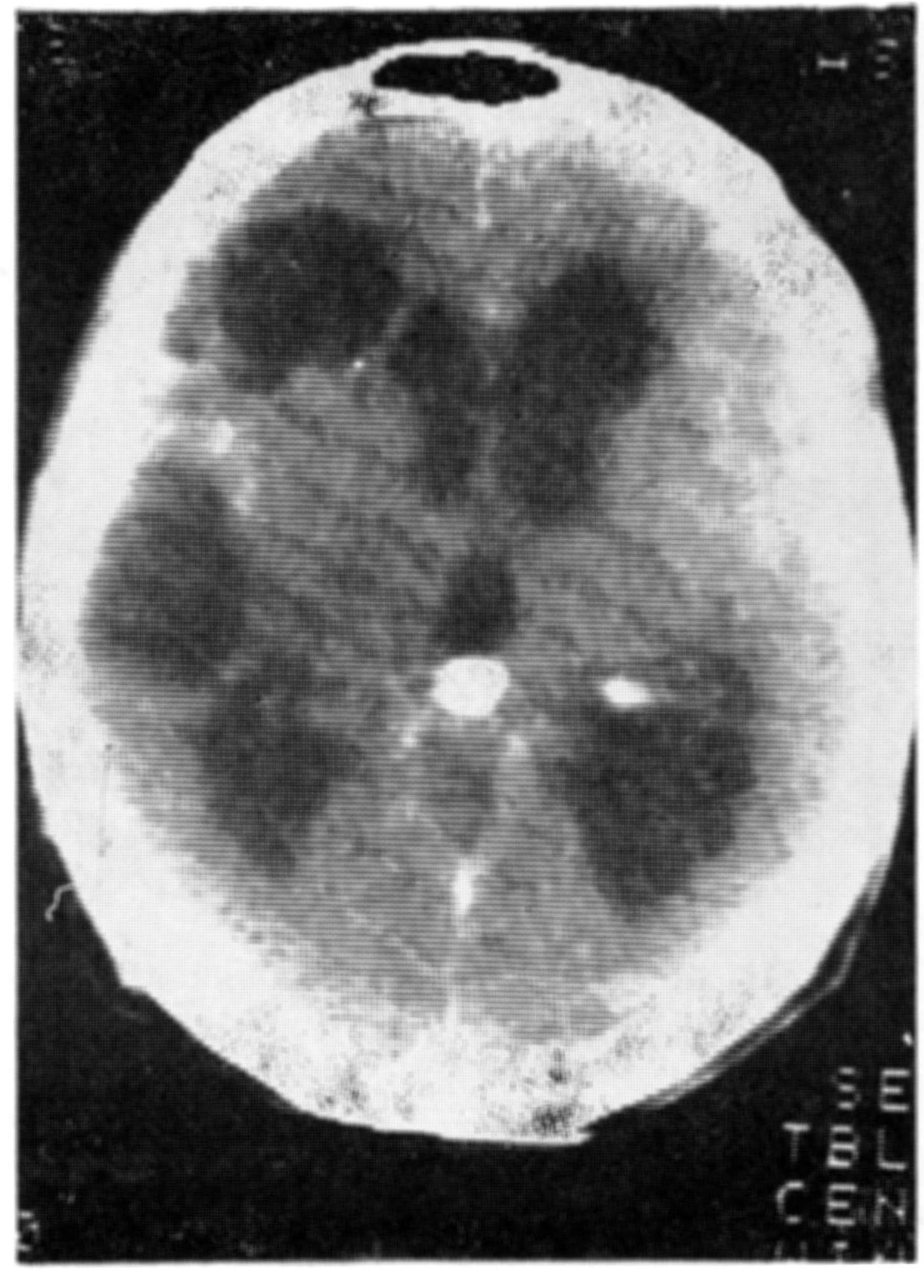

Fig. 1 - Tomografia computadorizada evidenciando áreas hipodensas no hemisfério cerebral direito, com discreta retencão de contraste na periferia, dilatacão ventricular e alcificação da pineal.

Fig. 2 - Tomografia

computadorizada mostrando pequena calcificação na região parietal esquerda $e$ cateter de derivacão no ventriculo lateral esquerdo.

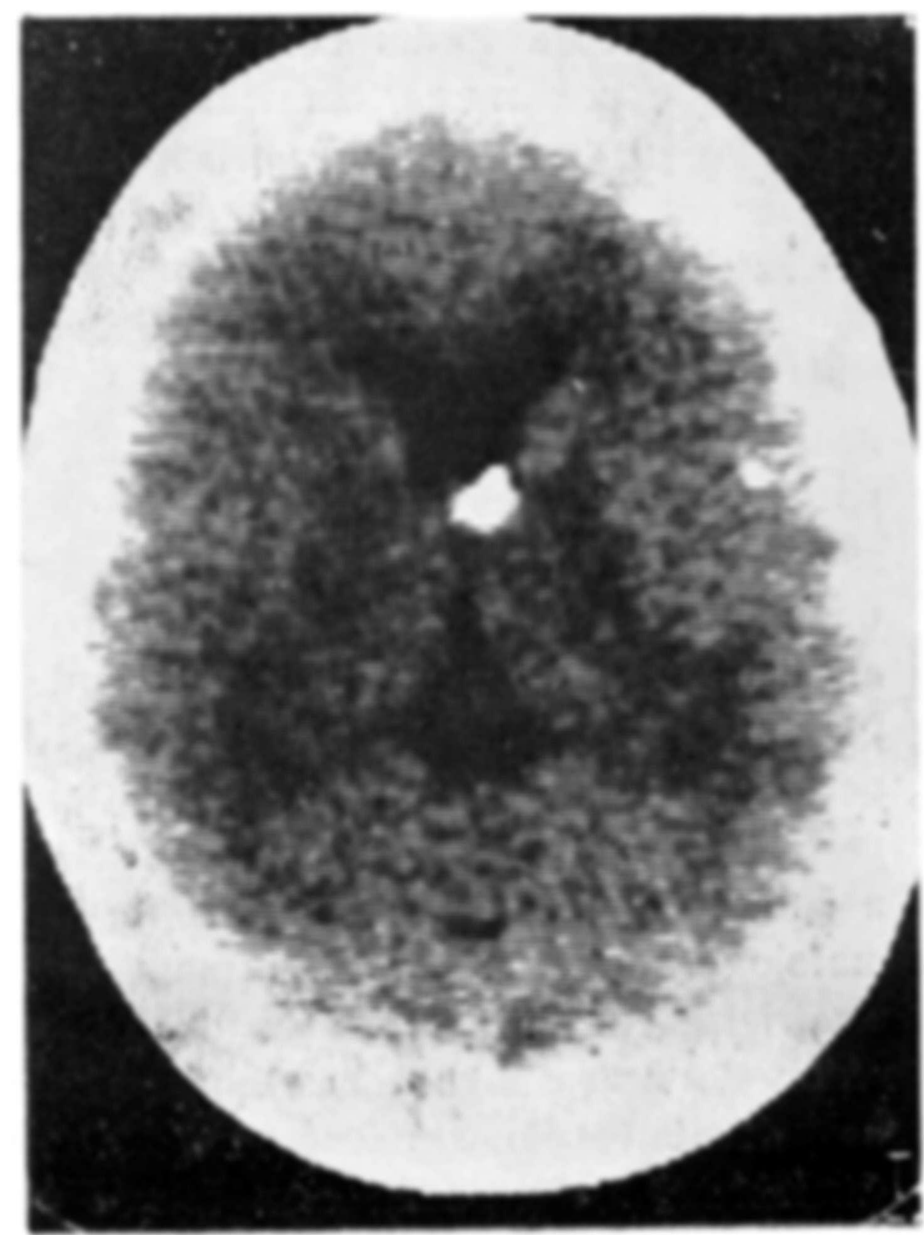




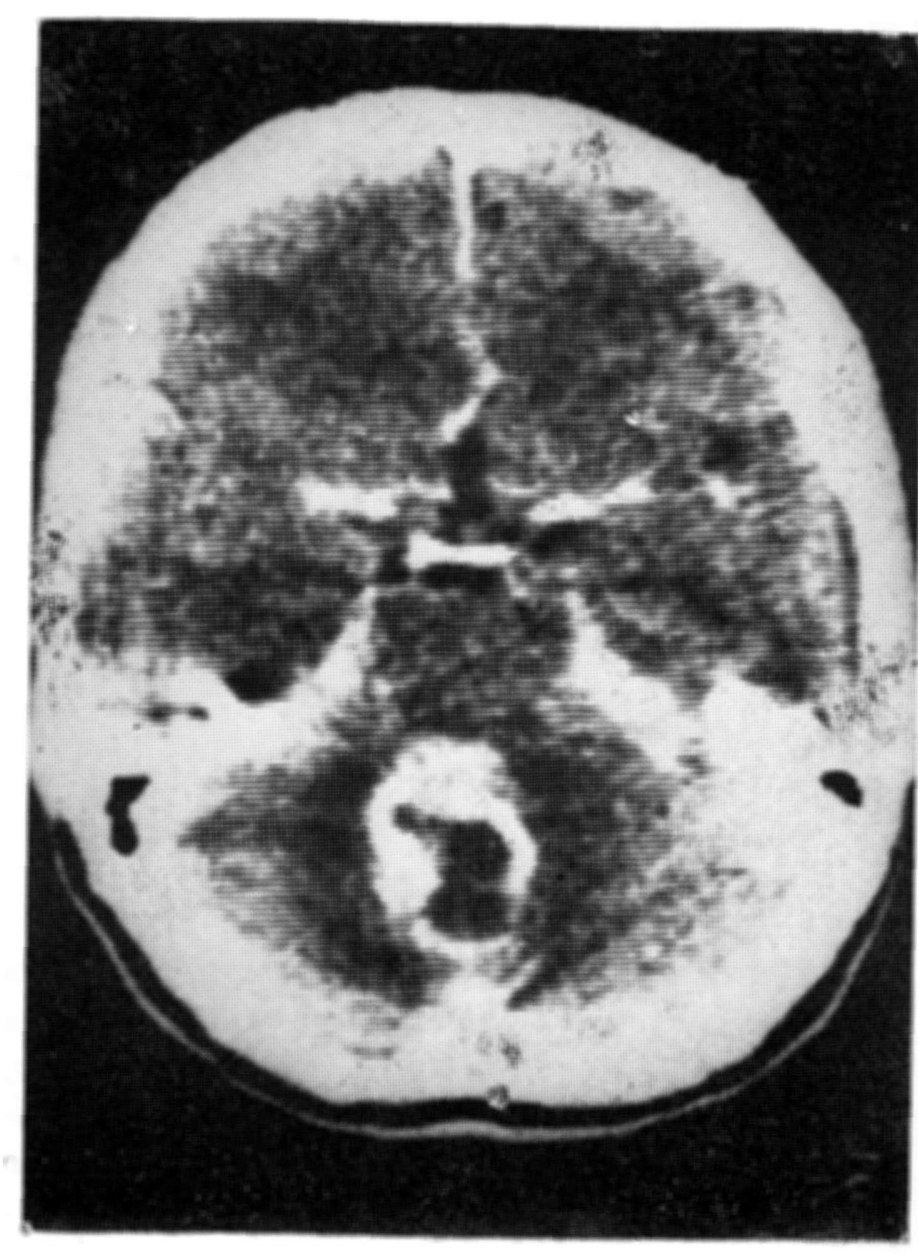

Fig. 3 - Tomografia computadorizada mostrando lesão cística ocupando a região do IV ventriculo $e$ vermis cerebelar, com retensão irregular do contraste na periferia e septaçoes.

\section{COMENTARIOS}

As alterações observadas nas TC dos pacientes com hidrocefalia por neurocisticercose foram compativeis às de outros autores 7,8,11,15,18,19,20,22,23,29, $31,34,36,39$ e confirmam, em vida, as observações anatomopatológicas de necrópsias que justificam o polimorfismo clínico da doença. As alterações do parênquima encefálico podem ser classificadas em ativas e cicatriciais. As lesões em atividade são representadas por cisto parenquimatosos, com ou sem áreas de captação de contraste, e significam que o parasita está vivo ${ }^{23,29}$. As lesões cicatriciais são representadas pelas calcificações, mais facilmente evidenciadas nas TC do que na radiografia simples do crânio, conforme já demonstrado por outros autores 7,23 , e que são os restos de involução do parasita após a sua morte. Preferimos as denominações de lesões ativas e cicatriciais ao invés de agudas e crônicas ${ }^{29}$, porque o tempo que decorre para que haja a morte e calcificação dos parasitas é bastante variável ( 1 a 10 anos) ${ }^{11,29}$ no mesmo paciente, possibilitando a coexistência de lesões ativas e cicatriciais num mesmo período. Da mesma forma, a determinação do tempo de evolução da doença pela manifestação clínica não é fiel, visto que dificilmente podemos precisar a época da infestação. Um dos nossos pacientes, que foi atendido com sintomatologia de HIC com 3 semanas de evolução, apresentava lesões ativas e cicatriciais na TC além de dilatação ventricular. A presença de edema ao redor de áreas hipodensas hipercaptantes (reação inflamatória do hospedeiro ao parasita 7,11,23) e o fenômeno de captação de contraste ao redor do parasita (membrana do parasita com reação inflamatória; reação glial e vasculite do 
tecido cerebral ao redor do parasita ${ }^{\mathbf{7 , 2 3 , 3 9}}$ ) indicam a existência de reação inflamatória do hospedeiro ao parasita. A ausência de captação ao redor dos cistos intraventriculares ou no espaço subaracnóideo demonstra a inexistência de reações inflamatórias; quando elas existem como relatado por Zee e col. ${ }^{39}$, provavelmente significam que o cisto está aderido ao epêndima e com reação glial ao seu redor, conforme observado em um dos nossos pacientes que apresentava cisto com halo captante no IV ventrículo (Fig. 3) e que durante a ressecção cirúrgica mostrava-se extremamente aderido ao tecido cerebral pela reação inflamatória. As alterações evidenciadas no sistema ventricular foram inespecíficas e contribuiram pouco para o diagnóstico etiológico da neurocisticercose. A dilatação ventricular foi observada constantemente e, em apenas um caso, verificamos captação de contraste ao redor e no interior de massa cística que ocupava o IV ventrículo, no qual o diagnóstico foi confirmado por exame anatomopatológico da peça cirúrgica. Nos outros casos foi impossivel a determinação de cisticercos no sistema ventricular, devido à ausência de captação, e à semelhança dos índices de absorção do líquido do interior dos cisticercos e do LCR. Especificamente, o grupo de pacientes estudados, seria classificado como de cisticercose da fossa posterior devido às manifestações de HIC por hidrocefalia. Com o advento da TC demonstrou-se claramente, através da identificação de cistos parenquimatosos em diferentes fases de evolução, o quanto esta classificação baseada em critérios clínicos é vulnerável do ponto de vista anatomopatólógico, conforme destacado anteriormente 13.

A comparaç̧ão entre as ventriculografias e as TC realizadas na mesma época, evidenciou que a ventriculografia fornece melhores informações sobre o sistema ventricular. Destacam-se entre estas a presença de hidrocefalia, os locais e as características morfológicas da obstrução, que algumas vezes são muito sugestivas da presença de um cisticerco. Pode demonstrar também cisticercos intraventriculares, móveis ou não, e reações inflamatórias nas paredes dos ventrículos. Por outro lado, a TC fornece importantes informaçōes sobre o parênquima encefálico.

A realização da TC com contraste positivo hidrossolúvel no interior dos ventrículos 23, 26 permite avaliação completa do parênquima e do sistema ventricular, com grande precisăo na determinação de cistos intraventriculares. Estes surgem como imagens negativas no interior dos ventrículos. $O$ uso deste contraste intraventricular permite ainda a realização da ventriculografia previamente à TC, conforme temos feito recentemente.

O uso da TC e da ventriculografia, possibilitando a definição do quadro encefálico quanto à presença e efeitos dos cisticercos no parênquima e no sistema ventricular, permite que possamos optar mais racionalmente por tratamentos cirúrgicos mais simples, como as derivações do LCR, ou mais complexos, como as exéreses de cisticercos ou ainda por ambos. As derivações do LCR estariam indicadas nos casos de hidrocefalia obstrutiva causada por processos inflamatórios ou pela presença de cisticercos, único ou múltiplos, acompanha- 
dos por reações inflamatórias e sem efeito de massa. As cirurgias para exérese de cisticercos, mesmo no interior dos ventrículos, estariam indicadas nos casos em que o cisticerco esteja exercendo efeito de massa, ou seja único e que não tenha desencadeado reação inflamatória (cisticerco livre intraventricular) ou quando sua presença, aliada ou não a processos inflamatórios, determine obstrução dentro do próprio ventrículo lateral (buraco de Monro, "carrefour" ventricular). A associação dos dois procedimentos terapêuticos está indicada nos casos em que se deseja o alívio imediato da hipertensão intracraniana devida à hidrocefalia, cuja abordagem direta não surtiu efeito satisfatório quanto à desobstrução das vias liquóricas.

Dentro do que foi analisado é possivel considerar as conclusões que se seguem. Apesar do diagnóstico de certeza da neurocisticercose em vida ainda só pode ser feito mediante a positividade das reações antígeno-anticorpo no LCR ou da observação cirúrgica, a TC propiciou novas perspectivas para o diagnóstico radiológico da neurocisticercose, mostrando alterações muito sugestivas das doenças, principalmente considerando-se pacientes de áreas endêmicas. A TC juntamente com a ventriculografia e, ainda melhor, a TC com contraste positivo no interior dos ventrículos, constituem-se nos exames mais importantes na avaliação completa do parênquima encefálico e do sistema ventricular, nesta patologia que atinge difusamente o sistema nervoso central. A avaliação global dos efeitos do parasita no encéfalo permite indicação mais racional das possiveis terapêuticas cirúrgicas (derivações do LCR e abordagens diretas para exérece de cisticercos. A TC permite ainda o acompanhamento da evolução da doença em suas diferentes fases, determinando a atividade ou não dos parasitas no encéfalo.

\section{RESUMO}

Estudo das alterações observadas nas tomografias computadorizadas (TCs) de 16 casos de neurocisticercose, com hipertensão intracraniana por hidrocefalia. Todos os casos foram submetidos a radiografias simples do crânio e 12, à ventriculografia com Dimer-X. Nas radiografias simples foram observados sinais de hipertensão intracraniana em 7 , calcificações em um, e 8 foram normais. A TC mostrou parenquima cerebral normal em 9 casos e nos 7 restantes áreas hipodensas arredondadas de tamanho variado, com ou sem retenção de contraste na periferia, e/ou calcificações esparsas no parênquima. Hidrocefalia foi a única alteração observada no sistema ventricular nos casos em que o exame foi realizado previamente à derivação do LCR. As ventriculografias foram analisadas em trabalho anterior e permitiram localizar e determinar as características da obstrução e a presença de massas ocupando espaço dentro dos ventrículos. A comparação das ventriculografias e TCs realizadas na mesma época evidenciou que a primeira fornece melhores informações sobre o sistema ventricular mas, por outro lado, a tomografia fornece informações adicionais sobre o parênquima cerebral. A TC e a ventriculografia são exames que se completam na avaliação e no diagnóstico destes pacientes. 
SUMMARY

Computorized tomography in patients with increased intracranial pressure and obstructive hydrocephalus due to cerebral cysticercosis: comparison with Dimer-X ventriculography

The authors reviewed the alterations observed on computorized tomography (CT) examinations of 16 patients with increased intracranial pressure and obstructive hydrocephalus due to cerebral cysticercosis. Plain radiograms of the skull were available in all cases and Dimer-X ventriculography in 12 cases. In 7 cases there were radiologic signs of increased intracranial pressure. CT scan showed normal cerebral parenchyma in 9 cases and low density areas, with various size, with or without surround contrast enhancement, and/or small calcifications in the parenchyma. Hydrocephalus was the only alteration observed in the ventricular system in the case that the examination was done before ventricular drainage. Ventriculography was analyzed in other paper and permitted to situate the obstruction, determine its morphological characteristics and identify occuping space lesions within the ventricles. Comparison between ventriculography and CT scan made in the same period evidenced that the former gives best information about the ventricular system and that tomography gives additional information about the cerebral parenchyma. In conclusion, CT scan and ventriculography are investigations that complete each the other for evaluation and diagnosis of cerebral cysticercosis.

\section{REFHRENCIAS}

1. ALMfIDA, G. M.; PEREIRA, W. C. \& FACURE, N. O. - Ventriculo-auriculostomia nos bloqueios ao trânsito do liquido cefalorraqueano na cisticercose encefálica. Arq. Neuro-Psiquiat. (São Paulo), 24:163, 1966.

2. ANTONOV, . P. \& KASTRITSKAYA, Z. M. - X-Ray diagnosis of cysticercosis of the brain. (Resumo em inglês). Vestn. Rentgenol. Radiol. 6:72, 1974.

3. ARANA, R. \& ASENJO, A. - Ventriculographic diagnosis of cysticercosis of the posterior fossa. J. Neurosurg. $2: 181,1945$.

4. ARSENI, C. \& CRITESCU, A. - Cisticercoza sistemului ventricular supratentorial. Rev. Med. inter (Neurol. Psihiatr.) 19:331, 1974.

5. ARserI, C. \& SAMITCA, D. C. - Cysticercosis of the brain. Brit. med. J. 5043:494, 1957.

6. ASENJO, A. - Setenta y dos casos de neurocisticercosis en el Instituto de Neurocirurgía. Rev. Neuropsiquiat. (Lima) 13:337, 1950.

7. BALAPARAMESWARARAO, S. \& DINAKAR, I. - Ventriculographic features of cerebral cysticercosis. Br. J. Radiol. 43:267, 1970.

8. BENTSON, J. R.; WILSON G. H.; HELMER, E. \& WINTER, J. - Computed tomography in intracranial cysticercosis. J. comp. Assist. Tomogr. 1:464, 1977.

9. BOTERO, D. \& CASTANO, S. - Treatment of cysticercosis with praziquantel in Colombia. Am. J. trop. Med. Hyg. 31:810, 1982.

10. CANELAS, H. M. - Neurocisticercose: incidência, diagnóstico e formas clínicas. Arq. Neuro-Psiquiat. (São Paulo), 20:1, 1962.

11. CARBaJaL J. R.; PALACIOS, E.; AZAR-KIA, B. \& CHURCHILL, R. - Radiology of cysticercosis of the central nervous system including computed tomography. Radiology 125:127, 1977.

12. CARDENAS $Y$ CARDENAS, J. - Cysticercosis. II. Pathologic and radiologic findings. J. Neurosurg. 19:635, 1962. 
13. COLLI, B. O. - Contribuição ao estudo do tratamento cirúrgico da neurocisticercose. Análise de 23 casos submetidos à derivação extracraniana (derivação ventrículo-atrial e ventriculo-peritoneal). Tese. Ribeirão Preto, 1979.

14. COLLI, B. O.; MARTELLI, N.; ASSIRATI JR., J. A. \& MAChADO, H. R. - Ventriculografia com Dimer-X em pacientes portadores de neurocisticercose com hipertensão intracraniana por hidrocefalia obstrutiva. Considerações sobre tratamento cirúrgico. No prelo.

15. DANZIGER, J. \& BLOCH, S. - Tapeworm cyst infestations of the brain. Clin. Radiol. 26:141, 1975.

16. DORFSMANN, J. - The radiologic aspects of cerebral cysticercosis. Acta radiol. $1: 836,1963$.

17. FACURE, N. O.; FACURE, J. J. \& NUCCI, A. - Aspecto tumoral da cisticercose intracraniana: abordagem cirúrgica. Arq. Neuro-Psiquiat. (Sø̃o Paulo) 36:200, 1978.

18. LAMAS, E. \& ESTEVEZ, J. - Dos casos de cisticercosis cerebral diagnosticados con tomografía axial computadorizada. Rev. clin. esp. 151:53, 1978.

19. LAMAS, E.; ESTEVEZ, J.; SOTO, M. \& OBRADOR, S. - Computerized axial tomography for the diagnosis of cerebral cysticercosis. Acta Neurochir. (Wien) 44:197, 1978.

20. LATOVITISKI, N.; ABRAMS, G.; CLARK, C.; MAYEUX, R.; ASCHERL JR., G. \& SCIARRA, D. - Cerebral cysticercosis. Neurology (Minneápolis), 28:838, 1978.

21. LEPE, A. \& CASTRO, M. - Radiologia de la cisticercosis cerebral. Neurocirurgía (Santiago) 19:191, 1961.

22. LOBATO, R. D.; LAMAS, E.; PORTILlO, J. M.; ROGER, R.; ESPARZA, J.; RIVAS, J. J. \& MUNOZ, M. J. - Hydrocephalus in cerebral cysticercosis. Pathogenic and therapeutic considerations. J. Neurosurg 55:786, 1981.

23. LOMBARDO, L. - La cisticercosis cerebral en México.. III. Diagnóstico. Gac. méd. mex 118:4, 1982.

24 - LOPES, P. G. - Contribuíção ao estudo do tratamento cirúrgico da cisticercose da fossa craniana posterior. Tese. Londrina, 1970.

25. LUYO, M.; KLERIGA, E. \& ESTANOL, B. - Fourth ventricular cysticercosis. Neurosurgery $7: 456,1980$.

26. MADRAZO, I.; RENTERIA, J. A. G.; PAREDES, G. \& OLHAFARAY, B. - Diagnosis of intravenetricular and cisternal cysticercosis by computerized tomography with positive intraventricular contrast medium. J. Neurosurg 55:947, 1981.

27. MARTELLI, N. - Tratamento cirúrgico da neurocisticercose. In F. C. PRADO, J. A. RAMOS \& J. R. VALLE (eds.) - Atualização Terapêutica. Ed. 12. Artes Médicas, São Paulo, pg 730.

28. MARTELLI, N.; COLLI, B. O.; ASSIRATI JR., J. A. \& MACHADO, H. R. Erosão óssea da base do crânio (regiões selar e para-selar) por cisticerco racemoso. Relato de caso. Arq. bras. Neurocirurg 1:273, 1982.

29. MERVIS B. \& LOTZ, J. W. - Computed tomography (CT) in parenchymatous cerebral cysticercosis. Clin. Radiol. 31:521, 1980.

30. OBRADOR, S. - Clinical aspects of cerebral cysticercosis. Arch. Neurol. Psychiat. 59:457, 1948.

31. PERCY, A. K.; BYRD, S .E. \& LOCKE, E. - Cerebral cysticercosis. Pediatrics 66:967, 1980.

32. PUPO, P. P.; REIS, J. B.; CARDOSO, W. \& SILVA, C. P. - Sobre a cisticercose encefálica. Estudo clínico, anátomo-patológico, radiológico e do líquido céfaloraqueano. Arq. Assis. psicop. São Paulo, 10-11:3, 1945/1946.

33. REDDY, D. G. \& RAMAMURTHY, B. - Ventriculographic changes in cysticercosis of the brain. Br. J. Surg. 41:11, 1953.

34. RODRIGUEZ, J. C.; GUTIGRREZ, R. A.; VALDES, O. D.; DORFSMAN, J. F. The role of computed axial tomography in the diagnosis and treatment of brain inflammatory and parasitic lesions: our experience in México. Neuroradiolgy 16: $458,1978$.

35. SANTIN, G. \& VARGAS, J. - Roentgen study of cysticercosis of central nervous system. Radiology 86:520, 1966. 
36. SHIBATA, M. K.; BIANCO, E.; MOREIRA, F. A. \& ALMEIDA, G. M. - Forma tumoral da cisticercose cerebral: diagnóstico pela tomografia computadorizada. Arq. Neuro-Psiquiat. (São Paulo) 38:399, 1980.

37. STEPIN, L. - Cerebral cysticercosis in Poland. Clinical symptons and operative treatment. J. Neurosurg. 19:505, 1962.

38. TAKayANAGUI, O. M. - Aspectos clínicos da neurocisticercose. Análise de 500 casos. Tese. Ribeirão Preto, 1980.

39. ZEE, C. S.; SEGALL, H. D.; MILLER, C.; TSAI, F. Y.; TEAL, J. S.; HIESHIMA, G.; AHMADI, J. \& HALLS, J. - Unusual neuroradiological features of intracranial cysticercosis. Radiology 137:397, 1980.

Departamento de Cirurgia, Ortopedia e Traumatologia (Disciplina Neurocirurgia) - HC Campus - 14100, Ribeirdo Preto, SP - Brasil 